\title{
Infrared Matrix-Assisted Laser Desorption and Ionization by Using a Tunable Mid-Infrared Free-Electron Laser
}

\author{
Rainer Cramer* and Franz Hillenkamp \\ Institut für Medizinische Physik und Biophysik, Westfälische/Wilhelms Universität, Münster, Germany
}

\author{
Richard F. Haglund, Jr. \\ Department of Physics and Astronomy and Free-Electron Laser Center for Biomedical and Materials Research, \\ Vanderbilt University, Nashville, Tennessee, USA
}

\begin{abstract}
Initial results of infrared matrix-assisted laser desorption/ionization (IR-MALDI) mass spectrometry of proteins by using the Vanderbilt free-electron laser as the source of selective vibrational excitation are reported. The ability of this laser to initiate desorption and ionization by excitation of specific vibrational modes is demonstrated. For the first time it is shown that IR-MALDI mass spectrometry at wavelengths other than those available from conventional fixed-frequency IR lasers, that is, 2.79 (Er:YSGG), 2.94 (Er:YAG), and 9.3-10.6 $\mu \mathrm{m}\left(\mathrm{CO}_{2}\right)$, is feasible and exhibits similar performance. IR-MALDI mass spectra were taken in the wavelength ranges 2.8-4 and 5.5-6.5 $\mu \mathrm{m}$, covering the absorption bands of the $\mathrm{O}-\mathrm{H}$ and $\mathrm{C}=\mathrm{O}$ stretch vibrations typical of many organic compounds such as succinic acid, fumaric acid, or nicotinic acid, which were used as matrices in these studies. A comparison between these results and Er:YAG/YSGG MALDI data are given. The potential of IR-MALDI at wavelengths near the $\mathrm{C}=\mathrm{O}$ stretch vibration and the possibilities for studies of the IR-MALDI mechanisms by using this kind of tunable source are discussed. (c) 1996 American Society for Mass Spectrometry (J Am Soc Mass Spectrom 1996, 7, 1187-1193)
\end{abstract}

$\mathrm{T}$ Together with electrospray ionization [1], matrixassisted laser desorption/ionization-mass spectrometry (MALDI-MS) $[2,3]$ is the most successful mass analysis technique for macromolecules in the mass range above $10^{4} \mathrm{Da}$. The most widely used matrix-assisted laser desorption/ionization (MALDI) techniques involve ultraviolet (UV) lasers and aromatic matrix materials. In the UV desorption and ionization process the matrix is believed to isolate the analyte molecules from each other and to donate or accept protons or cations to form even-electron ions, while shielding the analyte molecules from excessive vibrational or electronic energy transfer during desorption. By using UV-MALDI, proteins with masses in excess of $500,000 \mathrm{Da}$, carbohydrates, polymers, and oligonucleotides have been successfully analyzed, with sensitivities in the femtomolar range [4-7]. However, of the several hundred potential matrix materials described in the literature, fewer than 10 "good" UV

Address reprint requests to Rainer Cramer, Institut für Medizinische Physik und Biophysik, Robert-Koch-Strasse 31, 48149 Münster, Germany.

- Also at Department of Physics and Astronomy and Free-Electron Laser Center for Biomedical and Materials Research, Vanderbilt University, Nashville, TN. matrix materials are routinely used, and the search for new matrix materials is difficult and time consuming, in part because the correlation between matrix properties and MALDI mechanisms is not clearly understood [8-11].

In contrast, many potentially desirable matrix materials have richly structured mid-infrared absorption bands, which have been shown to be suitable for infrared-MALDI (IR-MALDI) [12]. For example, succinic acid, an aliphatic open chain carboxylic acid employed successfully in IR-MALDI, exhibits many strong absorption bands in its mid-infrared spectrum, for example, the $\mathrm{O}-\mathrm{H}$ and the $\mathrm{C}=\mathrm{O}$ stretch vibrations. The complex absorption spectra arising from these rovibronic degrees of freedom contrasts sharply with the simpler UV absorption spectrum of a typical aromatic matrix material, a spectrum often consisting of only a single relatively broad peak within the wavelength range accessible by conventional lasers. In addition, liquid matrix materials may be used in the infrared, whereas only a few, relatively ineffectual liquid matrices are known for UV-MALDI. Were it not for the scarcity of appropriate infrared laser sources, the spectral richness of the vibrational infrared region would surely make IR-MALDI appear an exciting and versa- 
tile analytical technique for determining the masses of oligonucleotides $[5,13]$ or proteins in electro-blotted gels [14].

In this article initial MALDI results over the wavelength range 2.8-4 and 5.5-6.5 $\mu \mathrm{m}$ acquired with a tunable infrared free-electron laser (FEL) are reported. First data on IR-MALDI performance by using a FEL and a comparison to the commonly used IR-MALDI with Er:YAG and TEA-CO 2 lasers and to UV-MALDI are also presented.

\section{Experimental}

The principal laser used in these experiments was the Vanderbilt mid-infrared free-electron laser (FEL). The free-electron laser, first demonstrated by Stanford researchers 15 years ago [15], has long been recognized as having potentially valuable applications in both materials science [16] and in medicine [17]. The Vanderbilt free-electron laser is based on the Mark III design originally demonstrated by Madey and his collaborators at Stanford [18]; substantial modifications and upgrades have improved both its reliability and the quality of the optical beam.

The FEL amplifies-by a purely classical effect-light emitted by a relativistic electron beam moving through a spatially periodic magnet array called a wiggler. The fundamental principles of freeelectron laser operation have been surnmarized in a number of review papers [19-21]. Unlike a conventional laser, the wavelength of the FEL is controlled by the wiggler period and the electron-beam energy, while the temporal pulse structure is determined by the type of accelerator structure chosen for the machine. In the case of the experiments reported here, the FEL was tuned over the range $2.8-6.5 \mu \mathrm{m}$ with energies from 20 to $60 \mathrm{~mJ}$ in a $4-\mu \mathrm{s}$ macropulse.

These unusual characteristics of the FEL alleviate some of the problems with current "table-top" infrared lasers. The interpretation of previous experiments with line-tunable light $(9.3-10.6 \mu \mathrm{m})$ from a transversely excited atmospheric (TEA) $\mathrm{CO}_{2}$ laser, for example, is seriously limited by variations in temporal structure and spatial intensity profile of the laser pulse at the target. In a TEA- $\mathrm{CO}_{2}$ laser, gain varies strongly with wavelength, the age of the gas mixture, and the mode structure of the laser. The laser pulse has a long (up to a few microseconds) tail following the typically 100-200-ns peak of the gain-switched pulse, unless a special gas mixture is used at the expense of output energy. While this tail has a much lower intensity than the peak, it may have an equal amount of energy. On low-gain laser lines, this tail cannot be suppressed even by changing the laser gas mix. Thus even relatively small wavelength variations can alter other essential characteristics of the $\mathrm{CO}_{2}$ laser pulse, making it difficult to compare data acquired at one wavelength with those at another. All of these problems either can be eliminated or substantially reduced with the freeelectron laser.

The Vanderbilt FEL delivers a nominal 4- $\mu$ s macropulse at a pulse repetition frequency of up to 30 $\mathrm{Hz}$; the macropulse comprises about $10^{4}$ micropulses that are 1 ps in duration and separated by intervals of 350 ps. The spatial pulse structure is a clean TEM T0 $_{00}$ mode as it comes from the resonator, but the long distance between resonator and target (some 25-30 m) creates some distortion in the beam, since there are many optical surfaces in the evacuated beam-transport system. The tuning range of the Vanderbilt FEL includes the $\mathrm{O}-\mathrm{H}$ and $\mathrm{N}-\mathrm{H}$ stretching vibrations around $3 \mu \mathrm{m}$, as well as many other vibrational bands in the fingerprint region out to $8 \mu \mathrm{m}$. Tables 1 and 2 summarize the accelerator operating parameters and optical characteristics of the Vanderbilt free-electron laser, respectively.

The mass spectrometer used for the investigations is a modified Comstock (Oak Ridge, TN) model TOF101-MS linear time-of-flight (TOF) mass spectrometer equipped with a microchannel plate detector. It consists of a 1-m TOF tube and a turbomolecular-pumped vacuum chamber with a base pressure of $10^{-8}$ torr. By utilizing an air lock for fast sample insertion this pressure can be restored within a few minutes after changing the sample.

The modifications include a new two stage focusing ion source, shown in the inset of Figure 1, which permits the application of high voltage to both the target and the extraction electrode. Typical operating parameters are $27-30 \mathrm{kV}$ for the target, $20 \mathrm{kV}$ for the extraction electrode, and $0 \mathrm{kV}$ for the time-of-flight tube. An ion guide wire was also installed but not used, since it did not show any significant improvement compared with the sole use of the described ion

Table 1. Design and operating characteristics of the accelerator/free-electron laser

\begin{tabular}{lclc}
\hline \multicolumn{2}{c}{ Wiggler characteristics } & \multicolumn{2}{c}{ Electron-beam characteristics } \\
\hline \hline Wiggler type & Tapered & Electron-beam energy & $20-45 \mathrm{MeV}$ \\
Wiggler length & $108 \mathrm{~cm}$ & Micropulse current & $20-40 \mathrm{~A}$ \\
Wiggler period & $2.3 \mathrm{~cm}$ & Macropulse current & $250 \mathrm{~mA}$ \\
Maximum wiggler field & $0.44 \mathrm{~T}$ & Energy spread & $0.5 \%$ \\
Typical tuning time $( \pm 10 \%)$ & $5 \mathrm{~min}$ & Micropulse repetition rate & $2.865 \mathrm{GHz}$ \\
& & Normalized emittance & $4 \pi \times 10 \pi \mathrm{mm} / \mathrm{mrad}$ \\
\hline
\end{tabular}


Table 2. Optical characteristics of the free-electron laser

\begin{tabular}{lcc}
\hline Optical characteristics & Best observed & $\begin{array}{c}\text { Values used in } \\
\text { experiments }\end{array}$ \\
\hline \hline Wavelength range (fundamental) & $2.0-9.5 \mu \mathrm{m}$ & $2.8-7.0 \mu \mathrm{m}$ \\
Linewidth $\Delta \nu / \nu$ & $0.7 \%$ & $2-4 \%$ \\
Pulse-to-pulse timing jitter & $0.1 \%$ & $0.4 \%$ \\
Macropulse energy & $360 \mathrm{~mJ}$ & $20-60 \mathrm{~mJ}$ \\
Pulse-to-pulse energy stability & $5 \%$ & $10-15 \%$ \\
Macropulse length & $6 \mu \mathrm{s}$ & $4-5 \mu \mathrm{s}$ \\
Micropulse duration & $0.5 \mathrm{ps}$ & $1-2 \mathrm{ps}$ \\
Micropulse peak power & $2 \mathrm{MW}$ & $0.5-1 \mathrm{MW}$ \\
Pulse repetition frequency & $30 \mathrm{~Hz}$ & $20 \mathrm{~Hz}$ \\
Average power & $11 \mathrm{~W}$ & $2-3 \mathrm{~W}$ \\
\hline
\end{tabular}

source. Therefore it was maintained at the same potential as the time-of-flight tube for the experiments described here.

The experimental setup was designed to employ IR lasers as well as UV lasers both irradiating the sample at $45^{\circ}$ to the surface normal. By switching to the UV laser, it was possible to calibrate the mass spectrometer performance without sacrificing precious FEL beam time. Since this procedure can be done within seconds it was even possible to crosscheck the performance when the FEL was used. The entire experimental setup is shown schematically in Figure 1.

The UV side comprises a VSL-337ND nitrogen laser (Laser Science, Inc., Newton, MA), a continuously variable attenuating filter, a beam splitter to couple out a trigger signal, an $f=250-\mathrm{mm}$ focusing lens, and a dichroic mirror transmitting in the visible to enable the use of a video camera for sample observation.

The IR optical setup is somewhat more complex because a fast electrooptic switch (Pockels cell) has to

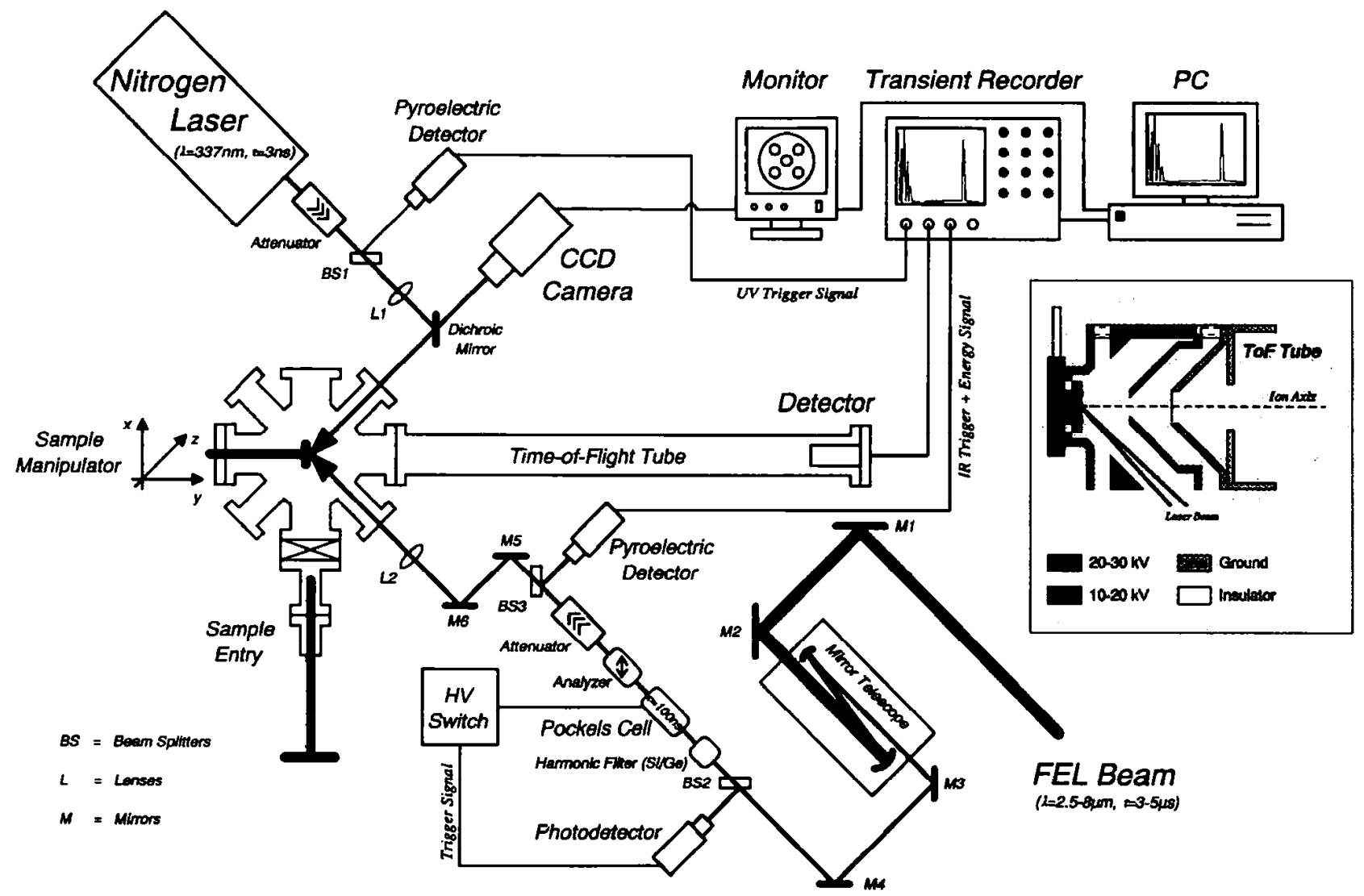

Figure 1. Experimental setup for both the free-electron laser and a nitrogen laser. The inset shows a schematic of the ion source. 
be used to slice a short micropulse train ( $\tau \geq 100 \mathrm{~ns}$ ) out of the FEL macropulse. Details of the Pockels cell have been reported elsewhere [22]. The first element in the optical train is a spherical mirror telescope, which decreases the beam diameter by a factor of 4 , so it can be accommodated by the free aperture of the Pockels cell. It is followed by a $\mathrm{BaF}_{2}$ beam splitter used to obtain a trigger signal for the Pockels cell driver electronics. Higher FEL harmonics ranging down to visible wavelengths, which could damage the CdTe crystal in the Pockels cell when high voltage is applied, are filtered out by a silicon window. After the Pockels cell, a polarizer consisting of $\mathrm{ZnSe}$ Brewster plates analyzes the time-dependent polarizations with a maximum extinction ratio of up to 1:200. Due to a residual depolarization of the beam, caused by the optical elements and unavoidable small misalignments of the Pockels cell, the actual suppression rate was measured to be typically $1: 160$. As a consequence, $\sim 80 \%$ of the total incident energy is contained in the $100-n s$ pulse. A second rotatable polarizer of the same type is used further down the optical train as an attenuator. Another $\mathrm{BaF}_{2}$ beam splitter provides the signal for monitoring the average energy of the individual irradiation pulses, as well as for triggering the transient oscilloscope. Finally an $f=85-\mathrm{mm}$ lens focuses the beam, producing an elliptical spot size on the target measuring approximately $250-350 \mu \mathrm{m}^{2}$.

The analog ion signal from the microchannel-plate detector is ac-coupled to a $10 \times$ preamplifier and digitized by a LeCroy (Chestnut Ridge, NY) $9310 \mathrm{M}$ transient recorder at time intervals of $10 \mathrm{~ns}$. The second channel of the transient recorder was also used to record the laser energy for each exposure simultaneously with the spectra as mentioned above. Further data processing was done with in-house software on a PC 486DX2.

All chemicals and analytes were purchased from Sigma Chemical Co. (St. Louis, MO) or Aldrich Chemical Co. (Milwaukee, WI) and used without further purification. If not stated otherwise, samples were prepared by mixing $1 \mu \mathrm{L}$ of a $10-\mu \mathrm{mol}$ analyte solution with 1-3 $\mu \mathrm{L}$ of a saturated matrix solution on the target and subsequently drying by means of a cold stream of air.

\section{Results and Discussion}

Initial experiments without the Pockels cell, that is, by using the full FEL macropulse, had already been successful with regard to obtaining MALDI spectra [23]. Spectra of proteins such as insulin (Figure 2) with succinic acid as matrix were obtained at several different wavelengths. As anticipated, these spectra exhibited poor mass resolution and small analyte-to-matrix signal ratio.

Cutting the duration of the FEL pulse to $100 \mathrm{~ns}$ with the Pockels cell dramatically improved mass resolution and raised the analyte-to-matrix signal ratio. At this

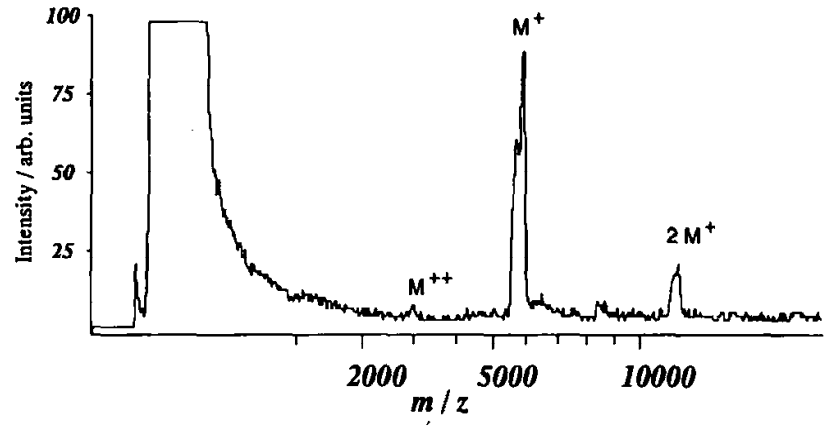

Figure 2. Insulin mass spectrum recorded with the full FEL macropulse; mass resolution $\leq 20$.

pulse width-typical for Er:YAG/YSGG or $\mathrm{CO}_{2}$ lasers used up to now in IR-MALDI-the protein spectra were recorded with mass resolutions at least 10 times better than those obtained with the full macropulse. Even bovine serum albumin along with its dimer could be detected, which was not possible with the 4- $\mu \mathrm{s}$-long macropulse. As a consequence of the superior performance with the shorter pulse, the spectra presented here were all taken by using the 100 -ns pulse. This also facilitated a comparison of the results with those obtained employing conventional IR lasers.

The first mass spectra recorded with the 100-ns short FEL pulse were taken at the Er:YAG wavelength of $2.94 \mu \mathrm{m}$. Several different matrices (fumaric acid, succinic acid, nicotinic acid) were used to record spectra of peptides with masses below $5 \mathrm{Da}$ (angiotensin I and II, substance $P$, renin substrate tetradecapeptide, melittin, glucagon, and chain B of insulin). As an example, Figure 3 shows a single-shot mass spectrum of the chain B of bovine insulin, in which the inset displays an expansion of the peptide ion region. In this mass range it is not difficult to obtain high mass resolution without any matrix signal. Furthermore it is possible to record peptide peaks with full width at half-maximum (FWHM) of only 30-40 ns and widths of only $50-70 \mathrm{~ns}$ at a signal intensity of $10 \%$ of the peak maximum. Hence it is evident that the ion formation is not maintained throughout the entire 100-ns

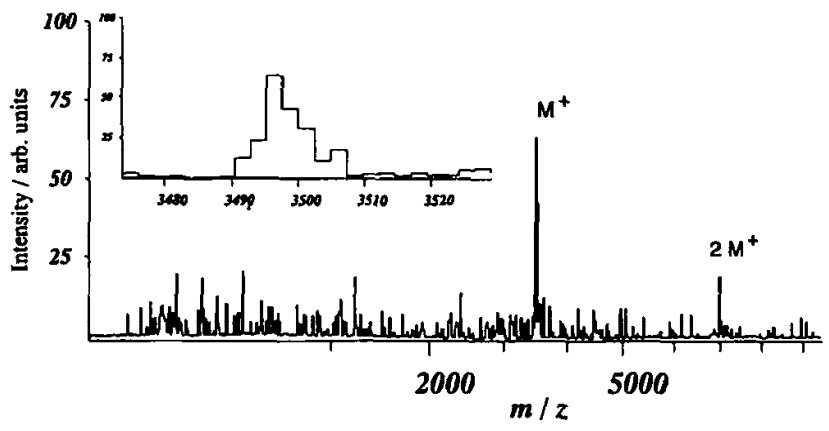

Figure 3. Single-shot spectrum of the chain B of insulin (matrix, nicotinic acid; wavelength, $2.94 \mu \mathrm{m}$; laser pulse width, $100 \mathrm{~ns}$; laser energy, $\sim 150 \mu \mathrm{J}$ ). 
pulse. This result also previously had been noted for matrix ions in IR-MALDI utilizing Er:YAG lasers [24].

Figure 4 shows a mass spectrum of bovine insulin with succinic acid as matrix at $2.94 \mu \mathrm{m}$. Ten successive single-shot mass spectra were accumulated from the same sample spot. In this case, a new sample preparation method was used. Acetone was added to the solvent and the sample was dried quickly in vacuo to achieve a reasonably homogeneous sample surface. The single-shot spectra of this sample reveal features similar to the peptide spectra mentioned above. The recorded FWHM of $20 \mathrm{~ns}$ is even smaller and corresponds to an apparent mass resolution of more than 900. Clearly, this mass resolution violates the sampling theorem for the sample rate used as well as the expected envelope function of the ${ }^{12} \mathrm{C} /{ }^{13} \mathrm{C}$ distribution. The sum spectrum shown in Figure 4 exhibits a more realistic mass resolution of approximately 500 which corresponds to a FWHM of 40 ns.

No significant difference can be seen between spectra recorded by using the FEL and those by using the nitrogen laser with the same instrument and settings. It is, however, easier with the FEL than with the nitrogen laser to obtain spectra without any matrix signals. A comparison of the IR-MALDI results by using the FEL with conventional IR-MALDI shows that they share a common feature: it is very difficult to obtain several spectra from the same sample spot, even though sample ablation was not as apparent as in the IR-MALDI utilizing Er:YAG lasers.

Studies of proteins in the mass range above $10 \mathrm{kDa}$ showed that MALDI-MS with the FEL possesses some advantages over UV-MALDI. With cytochrome $c$ as analyte the standard mass resolution for IR-MALDI was 400 to 500 , that is, a FWHM of $50 \mathrm{~ns}$ or $24 \mathrm{Da}$ and around 650 for best results, whereas a mass resolution of at best 200 could be obtained with the nitrogen laser. Figure 5 demonstrates that even for lysozyme it was possible to achieve a mass resolution of 600 . Additionally - as in the case of proteins with masses less than $10 \mathrm{kDa}$-matrix signals in the low mass range were often much less or even absent compared

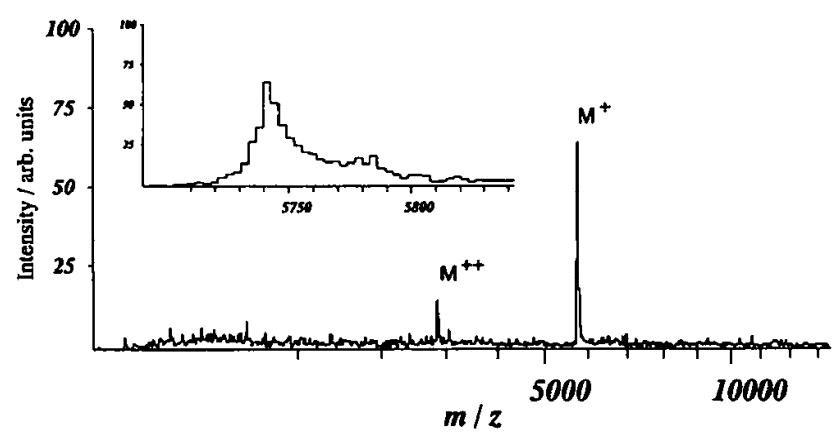

Figure 4. Sum spectrum of insulin, 10 successive single-shot spectra from the same sample spot were accumulated (matrix, succinic acid; wavelength, $2.94 \mu \mathrm{m}$; laser pulse width, $100 \mathrm{~ns}$; laser energy, $\sim 50 \mu \mathrm{J}$ ).

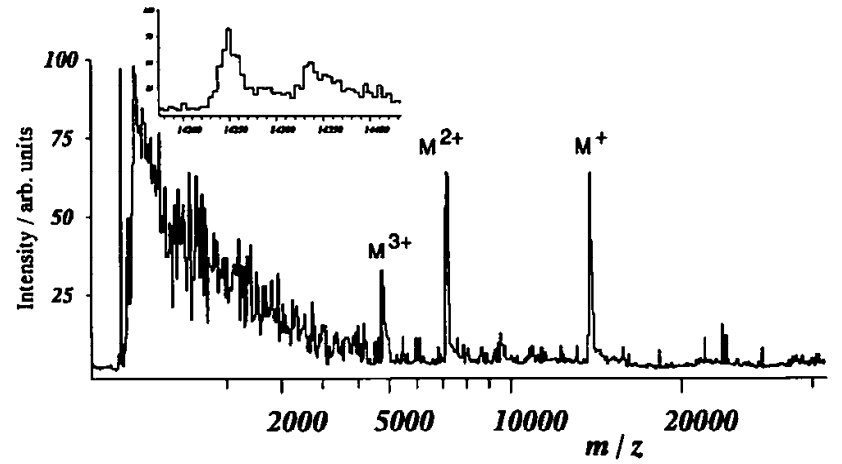

Figure 5. Single-shot spectrum of lysozyme (matrix, fumaric acid; wavelength, $2.94 \mu \mathrm{m}$; laser pulse width, $100 \mathrm{~ns}$; laser energy, $\geq 50 \mu \mathrm{J}$ ).

to UV-MALDI. On the other hand these results are in agreement with the "state of the art" performance of IR-MALDI utilizing reflectron-TOF mass spectrometry equipped with a secondary electron multiplier and an Er:YAG laser (Berkenkamp, S.; Menzel, C., private communication). In the case where succinic acid was used as the matrix, some of the spectra exhibited a peculiarly high degree of multiply charged molecule ions (Figure 6).

Mass spectra recorded at wavelengths other than $2.94 \mu \mathrm{m}$ are shown in Figures 7 and 8. Tuning the FEL toward these longer wavelengths near $3 \mu \mathrm{m}$ resulted in a degradation of the spectrum quality, that is, reduced mass resolution and analyte-to-matrix signal ratio, and an increase in the threshold energy necessary to obtain threshold spectra (Figure 7). The degradation of the mass resolution is mainly attributed to the increase of adduct ion formation, which depends on the matrix material used. Mass spectra obtained at wavelengths around $5.8 \mu \mathrm{m}$, close to the $\mathrm{C}=\mathrm{O}$ absorption, showed a similar performance to the $2.94-\mu \mathrm{m}$ spectra, yet the energy for threshold spectra was lower by a factor of 2 (Figure 8). These initial results at wavelengths far away from the broad $\mathrm{O}-\mathrm{H}$ absorption clearly show that IR-MALDI has potential at more than

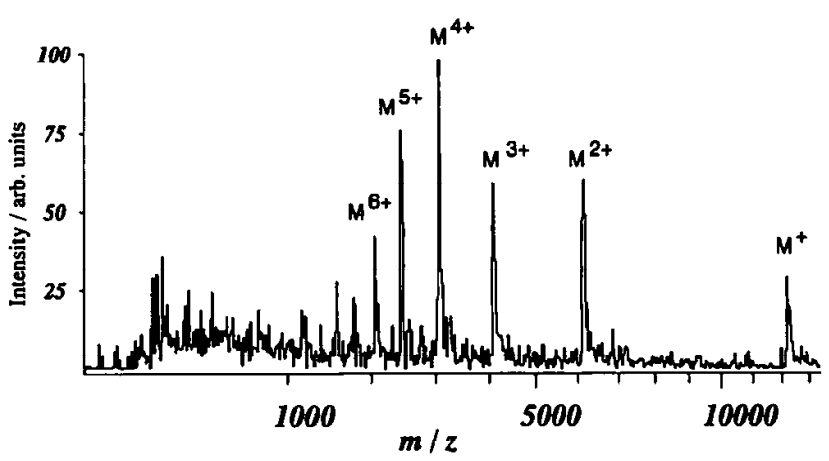

Figure 6. Single-shot spectrum of cytochrome $c$ (matrix, succinic acid; wavelength, $2.94 \mu \mathrm{m}$; laser pulse width, $100 \mathrm{~ns}$; laser energy, $\sim 50 \mu \mathrm{J}$ ). 


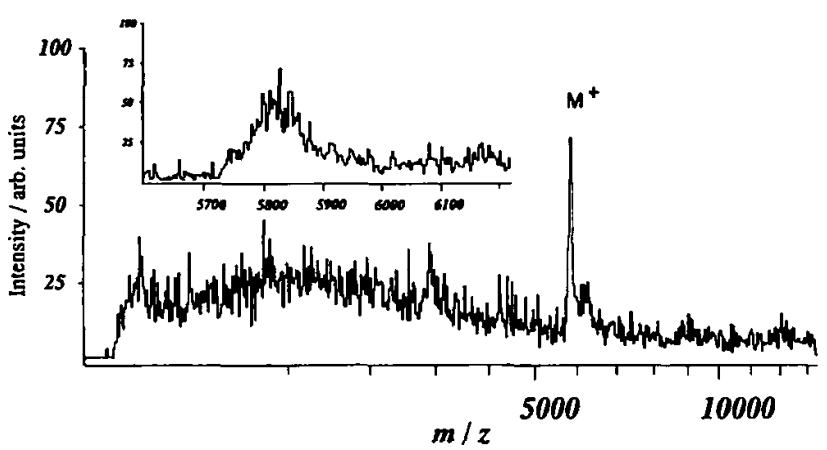

Figure 7. Single-shot spectrum of insulin (matrix, succinic acid; wavelength, $3.7 \mu \mathrm{m}$; laser pulse width, $100 \mathrm{~ns}$; laser energy, $\sim 400 \mu \mathrm{J})$.

one wavelength and more than one vibrational excitation mode, but this is a subject for further investigation.

Although it is true that the FEL is too big, too expensive, and too complex to be viewed as a routine analytical light source, it can be used to systematically study MALDI mechanisms by independently changing essential desorption and ionization parameters, and consequently the best set of parameters for a given analytical problem can probably be found. Once suitable protocols in pulse duration, wavelength, and so forth have been determined, it will be quite possible that other infrared light sources better suited to the table-top laboratory environment could be developed. Tunable infrared light can be generated by using optical parametric oscillator (OPO) systems or by stimulated electronic Raman scattering (SERS) [25-27] of superheated cesium vapor in a heat-pipe oven, pumped in either case by conventional nano- or picosecond Nd:YAG laser-pumped dye lasers. The latter can be tuned in a wide wavelength range $(3-11 \mu \mathrm{m})$ by changing dyes and solvents and by utilizing varying atomic transitions in different alkali metal vapors, yielding a broadly tunable high power (as much as a few millijoules) infrared light source. It should be noted, however, that the conversion efficiency of this scheme as a function of metal vapor species, pressure, temperature, heat-pipe design, optical alignment, and pump quality are all issues which have yet to be

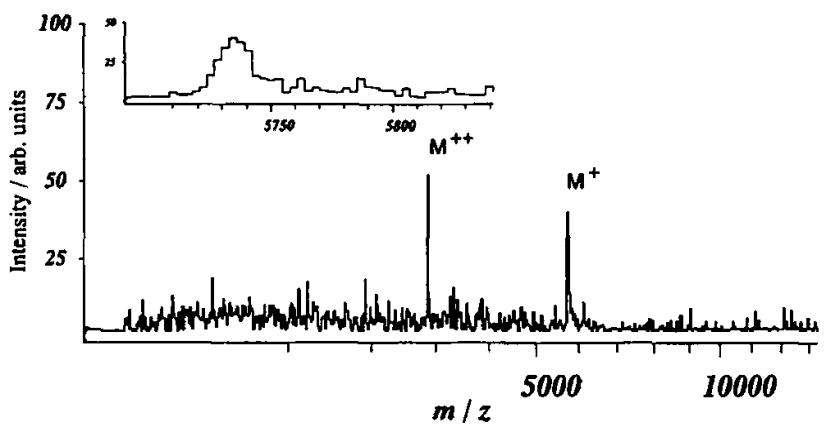

Figure 8. Single-shot spectrum of insulin (matrix, succinic acid; wavelength, $5.5 \mu \mathrm{m}$; laser pulse width, $100 \mathrm{~ns}$; laser energy, $\sim 50 \mu \mathrm{J})$. addressed systematically, whereas OPO systems are already commercially available in a wavelength region of up to $4 \mu \mathrm{m}$.

\section{Conclusions}

To summarize, it has been shown that the Vanderbilt free-electron laser, a tunable mid-infrared laser, is in spite of-or perhaps because of -its unusual temporal pulse structure suitable for generating high quality mass spectra over a broad wavelength range in the vibrational infrared portion of the spectrum. Masses as high as $66,000 \mathrm{Da}$ in the parent-ion peak and an excellent resolution of more than 500 for proteins like cytochrome $c$ and lysozyme with standard IR-MALDI sensitivities were observed. It was also shown that the IR-MALDI performance utilizing a linear TOF system equipped with a microchannel plate detector in combination with this special laser source is comparable to the standard Er:YAG IR-MALDI with a reflectron TOF system. Moreover it was shown that IR-MALDI is not only possible by using wavelengths close to the strong $\mathrm{O}-\mathrm{H}$ absorption band, but also in other vibrational absorption bands such as the $\mathrm{C}=\mathrm{O}$ stretch vibration at $6 \mu \mathrm{m}$.

All these results suggest that IR-MALDI experiments with the FEL permit the study of specific desorption and ionization routes, leading to a deeper understanding of the mechanisms of MALDI and opening the door to studying the potential applications of IR-MALDI in analytical technology. Therefore, future work will concentrate on systematic investigations of the wavelength and pulse length dependence of IR-MALDI by tuning the FEL and the Pockels cell independently and also on the development of alternative sources of tunable infrared radiation.

\section{Acknowledgments}

We would like to thank the FEL operator team and Jerri Tribble for their assistance with the FEL and the Pockels cell. This research was supported by the Medical Free-Electron Laser Program administered by the Office of Naval Research under Contract N-00014-91-C-0109. Rainer Cramer was supported by the German Academic Exchange Service (DAAD) through a doctoral stipend from funds of the HSPII/AUFE. This work is part of Rainer Cramer's research for his Ph.D. at the University of Münster.

\section{References}

1. Smith, R. D.; Loo, R. R. O.; Busman, M.; Udseth, H. R. Mass Spectrom. Rev. 1991, 10, 359-451.

2. Hillenkamp, F.; Karas, M.; Beavis, R. C.; Chait, B. T. Anal. Chem. 1991, 63, 1196A-1203A.

3. Bahr, U.; Karas, M.; Hillenkamp, F. Fresenius J. Anal. Chem. 1994, 348, 783-791.

4. Strupat, K.; Karas, M.; Hillenkamp, F. Int. J. Mass Spectrom. Ion Processes 1991, 111, 89-101.

5. Nordhoff, E.; Ingendoh, A.; Cramer, R.; Overberg, A.; Stahl, B.; Karas, M.; Hillenkamp, F. Rapid Commun. Mass Spectrom. $1992,6,771-776$. 
6. Vorm, O.; Roepstorff, P.; Mann, M. Anal. Chem. 1994, 66 , 3281-3287.

7. Jespersen, S.; Niessen, W. M. A.; Tjaden, U. R.; van der Greef, J.; Litborn, E.; Lindberg, U; Roeraade, J. Rapid Commun. Mass Spectrom. 1994, 8, 581-584.

8. Ehring, H.; Karas, M.; Hillenkamp, F. Org. Mass Spectrom. 1992, 27, 472-480.

9. Wu, K. J.; Steding, A.; Becker, C. H. Rapid Commun. Mass Spectrom. 1993, 7, 142-146.

10. Schneider, K.; Chait, B. T. Org. Mass Spectrom. 1993, 28, 1353-1361.

11. Fitzgerald, M. C.; Parr, G. R.; Smith, L. M. Anal. Chem. 1993, $65,3204-3211$.

12. Overberg, A.; Karas, M.; Bahr, U.; Kaufmarn, R.; Hillenkamp, F. Rapid Commun. Mass Spectrom. 1990, 4, 293-296.

13. Nordhoff, E.; Cramer, R.; Karas, M.; Hillenkamp, F.; Kirpekar, F.; Kristiansen, K.; Roepstorff, P. Nucl. Acids Res. 1993, 21, 3347-3357.

14. Strupat, K.; Karas, M.; Hillenkamp, F.; Eckerskorn, C.; Lottspeich, F. Anal. Chem. 1994, 66, 464-470.

15. Deacon, D. A. G.; Elias, L. R.; Maday, J. M. J.; Ramian, G. J.; Schwettman, H. A.; Smith, T. I. Phys. Rev. Lett. 1977, 38, 892-894.
16. Brau, C. A. Science 1988, 39, 1115-1120.

17. Danly, B. G.; Temkin, R. J.; Bekefi, G. IEEE J. Quantum Electron. 1987, QE-23, 1739-1750.

18. Benson, S. V.; Madey, J. M. J.; Schultz, J.; Marc, M.; Wadensweiler, W.; Westenskow, G. A.; Velghe, M. Nucl. Instrum. Methods Phys. Res. A 1986, 250, 39-43.

19. Sessler, A. M.; Vaughan, D. Am. Sci. 1987, 75, 34-43.

20. Freund, H. P.; Parker, R. K. Sci. Am. 1989, 260, 56-61.

21. Brau, C. A. Free-Electron Lasers; Academic Press: Boston, 1990.

22. Becker, K.; Johnson, J. B.; Edwards, G. Rev. Sci. Instrum. 1994, 65, 1496-1501.

23. Cramer, R.; Haglund, R. F., Jr.; Hillenkamp, F. Proccedings of the 43rd ASMS Conference on Mass Spectrometry and Allied Topics; Atlanta, GA, May 21-26, 1995; p 1063.

24. Overberg, A., dissertation, University of Münster, 1992.

25. Cotter, D.; Hanna, D. C.; Wyatt, R. Opt. Commun. 1976, 16, 256.

26. Harris, A. L.; Brown, J. K.; Berg, M.; Harris, C. B. Opt. Lett. $1984,9,47$

27. Hamadani, S. M.; Stockdale, J. A. D.; Compton, R. N. Pindzola. Phys. Rev. A 1986, 34, 1938. 